\title{
Toolbox for entanglement detection and fidelity estimation
}

\author{
Otfried Gühne, ${ }^{1}$ Chao-Yang Lu, ${ }^{2}$ Wei-Bo Gao, ${ }^{2}$ and Jian-Wei Pan ${ }^{2,3}$ \\ ${ }^{1}$ Institut für Quantenoptik und Quanteninformation, \\ Österreichische Akademie der Wissenschaften, 6020 Innsbruck, Austria \\ ${ }^{2}$ Hefei National Laboratory for Physical Sciences at Microscale and Department of Modern Physics, \\ University of Science and Technology of China, Hefei, Anhui 230026, People's Republic of China. \\ ${ }^{3}$ Physikalisches Institut, Universität Heidelberg, Philosophenweg 12, D-69120 Heidelberg, Germany
}

(Dated: October 28, 2018)

\begin{abstract}
The determination of the state fidelity and the detection of entanglement are fundamental problems in quantum information experiments. We investigate how these goals can be achieved with a minimal effort. We show that the fidelity of GHZ and W states can be determined with an effort increasing only linearly with the number of qubits. We also present simple and robust methods for other states, such as cluster states and states in decoherence-free subspaces.
\end{abstract}

PACS numbers: 03.65.Ud,03.67.Mn

Due to recent advances in quantum control many experiments aim at the generation and manipulation of multipartite quantum states $1,2,2,3$, 4]. In such an experiment, one typically aims at the creation of some pure entangled multi-qubit state $|\psi\rangle$, which can further be used for some quantum information processing task. Due to the unavoidable noise, however, the produced state will be some mixed state $\varrho_{\text {exp }}$, which may significantly differ from the desired state $|\psi\rangle$.

First, one may be interested in the fidelity of the produced state, that is, to what extent the desired state was prepared. This quantity is given by

$$
F_{\psi}=\left\langle\psi\left|\varrho_{\exp }\right| \psi\right\rangle
$$

and should ideally equal to one. In practice, however, it is lower, nowadays experiments with four or more qubits achieve typical fidelities between 0.5 and 0.9 .

Second, one may ask whether the prepared state was indeed genuine multipartite entangled. This means that the $N$-qubit state $\varrho_{\exp }$ shows entanglement effects which cannot be produced by $N-1$ qubits and all parties must have participated in the creation of the state $\varrho_{\exp }[5]$. To verify this criterion of success, entanglement witnesses can be used [3, 6, 7, 8, 9, 10, 11, 12]. These are observables which have by construction a positive expectation value on all unentangled (biseparable) states, a measured negative expectation value signals the presence of entanglement.

A generic witness for a pure state $|\psi\rangle$ is given by

$$
\mathcal{W}=\alpha \mathbb{1}-|\psi\rangle\langle\psi|
$$

where $\alpha$ is the maximal fidelity of $|\psi\rangle$ for biseparable states, a quantity which can be directly computed [3]. By construction, this observable is positive on the biseparable states. Furthermore, from the expectation value $\operatorname{Tr}\left(\varrho_{\exp } \mathcal{W}\right)$ we can determine the fidelity as $F_{\psi}=$ $\alpha-\operatorname{Tr}\left(\varrho_{\exp } \mathcal{W}\right)$, highlighting the close connection between fidelity determination and entanglement detection [13].

For the implementation, two questions are relevant. First, since quantum state tomography requires an exponentially increasing effort, the state $\varrho_{\exp }$ is often not completely known and one has to ask how many measurements are required for the evaluation of $\operatorname{Tr}\left(\varrho_{\exp } \mathcal{W}\right)$. Typically, only local measurements are possible, and an observable like

$$
\mathcal{M}=\sigma_{k} \otimes \sigma_{k} \ldots \otimes \sigma_{k}
$$

is called a local measurement setting $[3,6,8]$. Note that measuring all the coincidence probabilities of the $2^{N}$ possible outcomes of $\mathcal{M}$ gives also information about observables like $\mathbb{1} \otimes \sigma_{k} \ldots \otimes \sigma_{k}$, etc. A second question concerns the robustness to noise of the witness $\mathcal{W}$. As a simple model, one may consider the target state $|\psi\rangle$ mixed with white noise, $\varrho(p)=p|\psi\rangle\langle\psi|+(1-p) \mathbb{1} / 2^{N}$ and ask, how large $p$ has to be, in order that $\varrho(p)$ is detected by $\mathcal{W}$.

It has been shown in Ref. [9] that for important classes of states, namely GHZ states and cluster states, one can modify the witness in Eq. (2) such that it requires only two measurement settings and detects noisy states for $p>0.66$ (GHZ states), and this independently of the number of qubits. This shows that entanglement detection and fidelity estimation get not necessarily more difficult when increasing the number of qubits.

In this situation, three question are of interest. First, the question arises how a witness as in Eq. (2) can be implemented by local measurements, and how many measurements are needed. Second, the exact payoff between the robustness to noise of a witness and the number of required measurement settings is of interest. Finally, the question arises whether there are constructions of witnesses beyond the projector-based witness of Eq. (2).

In this paper we address all three questions. We first show how the fidelity of GHZ and W states can be determined with $N+1$ (resp. $2 N-1$ ) local measurements. Then, extending the results of Ref. [9] we derive witnesses for cluster states which are robust against noise and still require a small effort. Finally, we present a witness construction for the four-qubit singlet state, which is simpler to measure than the construction in Eq. (2), and, surprisingly, more robust against noise.

Let us start by discussing the local decomposition of 
the projector based witness for the case of GHZ states,

$$
\left|G_{N}\right\rangle=\frac{1}{\sqrt{2}}\left(|0\rangle^{\otimes N}+|1\rangle^{\otimes N}\right) .
$$

A witness for this state is given by $\mathcal{W}=\mathbb{1} / 2-\left|G_{N}\right\rangle\left\langle G_{N}\right|$ [3], with the term $\left|G_{N}\right\rangle\left\langle G_{N}\right|$ being decomposed as $2\left|G_{N}\right\rangle\left\langle G_{N}|=| 0\right\rangle\left\langle\left. 0\right|^{\otimes N}+\mid 1\right\rangle\left\langle\left. 1\right|^{\otimes N}+\mid 0\right\rangle\left\langle\left. 1\right|^{\otimes N}+\mid 1\right\rangle\left\langle\left. 0\right|^{\otimes N}\right.$. The terms $|0\rangle\left\langle\left. 0\right|^{\otimes N}\right.$ and $\left.\mid 1\right\rangle\left\langle\left. 1\right|^{\otimes N}\right.$ can be directly measured with the setting $\left(\sigma_{z}\right)^{\otimes N}$ since they correspond to eigenvectors of it. Using the fact that $|0\rangle\langle 1|=\left(\sigma_{x}+\right.$ $\left.i \sigma_{y}\right) / 2$ and $|1\rangle\langle 0|=\left(\sigma_{x}-i \sigma_{y}\right) / 2$ we can write the remaining term $\mathcal{X}=|0\rangle\left\langle\left. 1\right|^{\otimes N}+\mid 1\right\rangle\left\langle\left. 0\right|^{\otimes N}\right.$ as

$$
\mathcal{X}=\frac{1}{2^{N-1}} \sum_{k \text { even }}(-1)^{k / 2} \sum_{\pi} \bigotimes_{i=1}^{k} \sigma_{y} \bigotimes_{i=k+1}^{N} \sigma_{x}
$$

i.e. as an alternating sum of all products of the Pauli matrices $\sigma_{x}$ and $\sigma_{y}$ with an even number of $\sigma_{y}$. Here, $\sum_{\pi}$ denotes the sum over all permutations of the qubits, which yield different expressions.

Now we choose the $N$ measurement settings

$$
\mathcal{M}_{k}=\left[\cos \left(\frac{k \pi}{N}\right) \sigma_{x}+\sin \left(\frac{k \pi}{N}\right) \sigma_{y}\right]^{\otimes N}, \quad k=1, \ldots, N ;
$$

which are just measurements in the $x$-y-plane of the Bloch sphere with different angles, and obtain $\sum_{k=1}^{N}(-1)^{k} \mathcal{M}_{k}=N \mathcal{X}$ as can be checked by direct calculation [14].

Observation 1. For the determination of the fidelity of the $N$-qubit $G H Z$ state and evaluation on the witness $\mathcal{W}=\mathbb{1} / 2-\left|G_{N}\right\rangle\left\langle G_{N}\right|$ the $N+1$ measurement settings $\left(\sigma_{z}\right)^{\otimes N}$ and $\mathcal{M}_{k}$ are sufficient.

The required number of measurements can also be determined as follows: $\mathcal{X}$ in Eq. (5) contains in each term only an even number of $\sigma_{y}$ and $\mathcal{X}$ is symmetric under exchange of the qubits. The family of operators of this type is described by $\lfloor N / 2\rfloor+1$ parameters [15]. If we choose an arbitrary angle $\alpha_{k} \in(0, \pi / 2)$ and define $\mathcal{M}_{k}^{ \pm}=\left[\cos \left(\alpha_{k}\right) \sigma_{x} \pm \sin \left(\alpha_{k}\right) \sigma_{y}\right]^{\otimes N}$, then $\mathfrak{M}_{k}=\mathcal{M}_{k}^{+}+\mathcal{M}_{k}^{-}$ corresponds to two local measurements and also contains in each term only an even number of $\sigma_{y}$ and is symmetric under exchange of the qubits. Therefore, by choosing $\lfloor N / 2\rfloor+1$ different $\alpha_{k}$ we can express any observable of the former type as a linear combination of the $\mathfrak{M}_{k}$. Noting that we may also choose $\alpha_{k}=0$ corresponding to $\left(\sigma_{x}\right)^{\otimes N}$ (and, for $N$ even, also $\alpha_{k}=\pi / 2$ corresponding to $\left(\sigma_{y}\right)^{\otimes N}$ ), we see that any observable which contains in each term (in the Pauli matrix representation) only an even number of $\sigma_{y}$ (and $\sigma_{z}$ elsewhere) and is symmetric under exchange of qubits can be measured by $N$ local measurement settings. This argumentation also shows that the measurement directions are not unique.

In order to see how this helps for other states besides GHZ states, let us consider W states [16], defined by

$$
\left|W_{N}\right\rangle=\frac{1}{\sqrt{N}} \sum_{\pi}|0\rangle^{\otimes(N-1)}|1\rangle
$$

where $\sum_{\pi}$ denotes again the symmetrized version.

When decomposing $\left|W_{N}\right\rangle\left\langle W_{N}\right|$ into local measurements, the diagonal terms can again directly be measured with the $\left(\sigma_{z}\right)^{\otimes N}$ setting. The off-diagonal terms, can, using the fact that $|01\rangle\langle 10|+| 10\rangle\langle 01|=\left(\sigma_{x} \sigma_{x}+\sigma_{y} \sigma_{y}\right) / 2$ and $|0\rangle\langle 0|=\left(\mathbb{1}+\sigma_{z}\right) / 2$, be written as

$\mathcal{Y}=\frac{2^{1-N}}{N}\left(\sum_{\pi} \bigotimes_{i=1}^{2} \sigma_{x} \bigotimes_{i=3}^{N}\left(\mathbb{1}+\sigma_{z}\right)+\sum_{\pi} \bigotimes_{i=1}^{2} \sigma_{y} \bigotimes_{i=3}^{N}\left(\mathbb{1}+\sigma_{z}\right)\right)$

Each of the two terms in this sum contains only an even number of $\sigma_{x}$ (resp. $\left.\sigma_{y}\right)$ and a different observable $\left(\mathbb{1}+\sigma_{z}\right)$ elsewhere. Hence, as discussed before, we can measure each term with $N$ measurements settings of the type $\mathcal{M}_{k}^{ \pm}=\left[\left(\cos \alpha_{k} \sigma_{x / y} \pm \sin \alpha_{k}\left(\mathbb{1}+\sigma_{z}\right)\right]^{\otimes N}\right.$, which are effectively measurements in the $x$ - $z$-plane (resp. $y$ - $z$-plane) of the Bloch sphere. Using the fact that one of these settings can be chosen to be $\left(\mathbb{1}+\sigma_{z}\right)^{N}$, which is effectively a measurement of $\left(\sigma_{z}\right)^{N}$, we can summarize:

Observation 2. The fidelity of an $N$-qubit $W$ state can be determined by $2 N-1$ local measurements.

Consequently, also witnesses of the type $\mathcal{W}=\alpha \mathbb{1}-$ $\left|W_{N}\right\rangle\left\langle W_{N}\right|$ (and also the more general witnesses in Ref. [2]) can be measured with $2 N-1$ local measurements. Note that Observation 2 has general consequences in view of the results of Ref. [12]. There, it was shown that any pure multipartite entangled state can be brought by local operations close to the $\mathrm{W}$ state. Consequently, a witness of the type $\mathcal{W}=\alpha \mathbb{1}-\left|W_{N}\right\rangle\left\langle W_{N}\right|$, can, after reversing the appropriate local operations, detect any entangled pure state. Then, it is proved that the W state can be measured with $N^{2}-N+1$ settings, implying that any pure entangled state can be detected by $N^{2}-N+1$ measurements (although the robustness to noise may be small). Observation 2 shows that only $2 N-1$ measurements are already sufficient.

The optimal decomposition for the three-qubit W state with five measurements was already given in Ref. [8]. For the four-qubit case, we obtain the decomposition

$$
\begin{aligned}
& \left|W_{4}\right\rangle\left\langle W_{4}\right|=\frac{1}{64}\left(-2 \sum_{\pi} \mathbb{1} \mathbb{1} \mathbb{1} \sigma_{z}-4 \sum_{\pi} \mathbb{1} \mathbb{1} \sigma_{z} \sigma_{z}\right. \\
& -6 \sum_{\pi} \mathbb{1} \sigma_{z} \sigma_{z} \sigma_{z}-8\left(\sigma_{z}\right)^{\otimes 4}-2\left(\sigma_{x}\right)^{\otimes 4}-2\left(\sigma_{y}\right)^{\otimes 4} \\
& \left.+\sum_{\alpha=x, y}\left[\left(\mathbb{1}+\sigma_{z}+\sigma_{\alpha}\right)^{\otimes 4}+\left(\mathbb{1}+\sigma_{z}-\sigma_{\alpha}\right)^{\otimes 4}\right]\right)
\end{aligned}
$$

which requires the seven measurements of $\left(\sigma_{x}\right)^{\otimes 4}$, $\left(\sigma_{y}\right)^{\otimes 4},\left(\sigma_{z}\right)^{\otimes 4}$ and $\left(\sigma_{z} \pm \sigma_{\alpha}\right)^{\otimes 4}$ with $\alpha=x, y$.

Let us discuss cases, where even with a simple decomposition the determination of the fidelity requires too much effort. This may happen in multi-photon experiments, where the measurement of a setting like $\left(\sigma_{z}\right)^{\otimes N}$ may require a data collection time of several hours. In this case, instead of measuring the witness $\mathcal{W}=\alpha \mathbb{1}-|\psi\rangle\langle\psi|$ one can measure a witness like $\mathcal{W}^{\prime}=$ $\alpha \mathbb{1}-|\psi\rangle\langle\psi|+P$ where $P$ is a positive operator. Since 
$P$ has only positive eigenvalues, $\langle\mathcal{W}\rangle \geq 0$ implies that $\left\langle\mathcal{W}^{\prime}\right\rangle \geq 0$ so $\mathcal{W}^{\prime}$ is a valid witness, and allows to estimate the fidelity via $F_{\psi} \geq \alpha-\operatorname{Tr}\left(\mathcal{W}^{\prime} \varrho_{\exp }\right)$. The question is how to choose $P$ in order require only few measurements, while still obtaining a good bound on the fidelity.

Now we show how to derive a sequence of witnesses which are more and more robust to noise, while still being simple to implement. The idea is to start from a known witness of the type $\mathcal{W}^{\prime}=\alpha \mathbb{1}-|\psi\rangle\langle\psi|+P$ and to systematically subtract terms from $P$. We demonstrate this for the six-qubit cluster state, the techniques can be straightforwardly adapted to other graph states. The six-qubit cluster state is given by

$$
\left|C_{6}\right\rangle=\frac{1}{2}(|000000\rangle+|111000\rangle+|000111\rangle-|111111\rangle)
$$

This state is a graph state, corresponding after local rotations to the $\mathrm{H}$-shaped graph [4]. A typical witness for this state would be $\mathcal{W}=\mathbb{1} / 2-\left|C_{6}\right\rangle\left\langle C_{6}\right|$, however, the methods presented above yield a decomposition with 16 measurement settings. This witness detects a cluster state mixed with white noise for $p>31 / 63 \approx 0.492$.

In order to write down witnesses which require less effort, note that the cluster state can be described by its stabilizing operators [4, 17]. These are

$$
\begin{aligned}
& g_{1}=\mathbb{1} \sigma_{z} \mathbb{1}\left(\sigma_{x}\right)^{\otimes 3} ; g_{2}=\left(\sigma_{z}\right)^{\otimes 2}(\mathbb{1})^{\otimes 4} ; g_{3}=\mathbb{1}\left(\sigma_{z}\right)^{\otimes 2}(\mathbb{1})^{\otimes 3} ; \\
& g_{4}=\left(\sigma_{x}\right)^{\otimes 3} \mathbb{1} \sigma_{z} \mathbb{1} ; g_{5}=(\mathbb{1})^{\otimes 3}\left(\sigma_{z}\right)^{\otimes 2} \mathbb{1} ; g_{6}=(\mathbb{1})^{\otimes 4}\left(\sigma_{z}\right)^{\otimes 2} ;
\end{aligned}
$$

and $\left|C_{6}\right\rangle$ is the unique state fulfilling $g_{i}\left|C_{6}\right\rangle=\left|C_{6}\right\rangle$.

As the stabilizing operators describe the state, they can be used for the construction of witnesses. Indeed, as shown in Ref. [9], a witness is given by

$$
\tilde{\mathcal{W}}_{1}=3 \mathbb{1}-2\left(\prod_{i=1}^{3} \frac{g_{i}+\mathbb{1}}{2}\right)-2\left(\prod_{i=4}^{6} \frac{g_{i}+\mathbb{1}}{2}\right) .
$$

where the two groups of stabilizing operators correspond to the two-colourability of the H-shaped graph [10]. This witness requires only two measurement settings, namely $\left(\sigma_{z}\right)^{\otimes 3}\left(\sigma_{x}\right)^{\otimes 3}$ and $\left(\sigma_{x}\right)^{\otimes 3}\left(\sigma_{z}\right)^{\otimes 3}$. It detects states mixed with white noise for $p>0.7142$. In order to show that $\tilde{\mathcal{W}}$ is a witness one can directly calculate that $\tilde{\mathcal{W}}_{1}-2 \mathcal{W} \geq 0$, i.e., $\tilde{\mathcal{W}}_{1}=2 \mathcal{W}+P[9]$.

To improve this witness, let us look at $P=\tilde{\mathcal{W}}_{1}-2 \mathcal{W} \geq$ 0 . It is easy to see that $P / 2$ is a projector onto a 49 dimensional subspace. The idea is to find a product basis such that many vectors of this basis lie in this subspace. Then, these vectors can be measured with one setting and they can be subtracted from the witness. In our case, the desired basis turns out to be the computational basis, i.e., the eigenvectors of the measurement $\left(\sigma_{z}\right)^{\otimes 6}$.

Indeed, if we define for three qubits $\mathcal{A}=\mathbb{1}-$ $|000\rangle\langle 000|-| 111\rangle\langle 111|$ the operator $P-2 \mathcal{A} \otimes \mathcal{A}$ has no negative eigenvalues. Therefore, the observable

$$
\tilde{\mathcal{W}}_{2}=\tilde{\mathcal{W}}_{1}-2 \mathcal{A} \otimes \mathcal{A}
$$

is a valid witness, and requires three settings $\left[\left(\sigma_{z}\right)^{\otimes 3}\left(\sigma_{x}\right)^{\otimes 3},\left(\sigma_{x}\right)^{\otimes 3}\left(\sigma_{z}\right)^{\otimes 3}\right.$ and $\left.\left(\sigma_{z}\right)^{\otimes 6}\right]$ for its measurement. It detects states mixed with white noise for $p>0.579$, which is a reasonable improvement.

As a sidestep, note the interesting fact that the witness $\tilde{\mathcal{W}}_{2}$ may be improved by local filters. Such filters are invertible operators $F_{i}$ on each qubit, applying then $F=\bigotimes_{k=1}^{N} F_{k}$ to a state, $\varrho \mapsto F \varrho F^{\dagger}$, keeps the entanglement (or separability) of the state. For the case of complete knowledge of $\varrho$ it was demonstrated in Ref. 2], that one can alternatively transform a witness as $\mathcal{W} \mapsto F^{\dagger} \mathcal{W} F$, and optimize over $F$, in order to obtain a negative expectation value. Interestingly, if we restrict our attention to filters of the type $F_{i}=\alpha_{i}|0\rangle\left\langle 0\left|+\beta_{i}\right| 1\right\rangle\langle 1|$ the witness $F^{\dagger} \tilde{\mathcal{W}}_{2} F$ still needs the same three measurements as $\tilde{\mathcal{W}}_{2}$, showing that filtering can also be useful for the case of incomplete information.

In order to improve $\tilde{\mathcal{W}}_{2}$ further, we consider other eigenvectors of $P$, namely $\left|\phi_{1 / 2}\right\rangle=\left|v_{k}\right\rangle \otimes(|001\rangle \pm$ $|110\rangle) / \sqrt{2} ;\left|\phi_{3 / 4}\right\rangle=\left|v_{k}\right\rangle \otimes(|010\rangle \pm|101\rangle) / \sqrt{2}$ and $\left|\phi_{5 / 6}\right\rangle=$ $\left|v_{k}\right\rangle \otimes(|100\rangle \pm|011\rangle) / \sqrt{2}$, where $\left|v_{k}\right\rangle=|111\rangle\left(\left|v_{k}\right\rangle=\right.$ $|000\rangle)$ for $k$ odd (even) and the upper signs hold for odd $k$. Then we can define $\mathcal{P}_{1}=\sum_{i=1}^{6}\left|\phi_{i}\right\rangle\left\langle\phi_{i}\right|=$ $\mathcal{B} \otimes \mathcal{C}-\frac{1}{2}(\mathbb{1}-\mathcal{A}) \otimes \mathcal{A}$, where $\mathcal{A}$ is the same as before, $\mathcal{B}=|111\rangle\langle 111|-| 000\rangle\langle 000|$ and

$$
\mathcal{C}=\frac{1}{2 \sqrt{3}}\left[\left(\frac{\sqrt{3} \sigma_{x}+\sigma_{y}}{2}\right)^{\otimes 3}+\left(\frac{\sqrt{3} \sigma_{x}-\sigma_{y}}{2}\right)^{\otimes 3}\right] .
$$

So we arrive at the witness

$$
\tilde{\mathcal{W}}_{3}=\tilde{\mathcal{W}}_{2}-2 \mathcal{P}_{1}
$$

which requires five measurement settings, namely the three settings for $\tilde{\mathcal{W}}_{2}$ and the two settings for measuring $\mathcal{B} \otimes \mathcal{C}$, and tolerates noise as long as $p>0.543$. We can go further by considering six other vectors $\left|\phi_{k}\right\rangle$ for $k=7, \ldots, 12$, which arise form the $\left|\phi_{k}\right\rangle$ by swapping the qubits $1,2,3$ with the qubits $4,5,6$. For them, one can define a $\mathcal{P}_{2}$ similar to $\mathcal{P}_{1}$, and one arrives at the witness:

$$
\begin{aligned}
\tilde{\mathcal{W}}_{4} & =\tilde{\mathcal{W}}_{2}-2\left(\mathcal{P}_{1}+\mathcal{P}_{2}\right) \\
& =\tilde{\mathcal{W}}_{1}-\mathbb{1} \otimes \mathcal{A}-\mathcal{A} \otimes \mathbb{1}-2(\mathcal{B} \otimes \mathcal{C}+\mathcal{C} \otimes \mathcal{B})
\end{aligned}
$$

Note that for measurement of $\tilde{\mathcal{W}}_{4}$ the setting $\left(\sigma_{z}\right)^{\otimes 6}$ is not required anymore, so in total only six measurement settings are needed. The witness detects states for $p>$ 0.5 , so it is nearly as efficient as the original projector witness $\mathcal{W}=\mathbb{1} / 2-\left|C_{6}\right\rangle\left\langle C_{6}\right|$, however, $\tilde{\mathcal{W}}_{4}$ requires a significantly smaller effort. It is this witness, which has also been used in a recent experiment [4].

There is another feature which makes the witness $\tilde{\mathcal{W}}_{4}$ interesting from a general point of view. Namely, it can be written as

$$
\tilde{\mathcal{W}}_{4}=\frac{\mathbb{1}}{2}-\left|C_{6}\right\rangle\left\langle C_{6}|+| \tilde{C}_{6}\right\rangle\left\langle\tilde{C}_{6}\right|
$$

where $\left|\tilde{C}_{6}\right\rangle=(-|000000\rangle+|000111\rangle+|111000\rangle+$ $|111111\rangle) / 4$ is an orthogonal cluster state in a different 
basis. It is immediately clear that $\tilde{\mathcal{W}}_{4}$ is a witness, which is of a similar strength as the witness $\mathcal{W}=\mathbb{1} / 2-\left|C_{6}\right\rangle\left\langle C_{6}\right|$ since $\left|C_{6}\right\rangle$ and $\left|\tilde{C}_{6}\right\rangle$ are orthogonal. Also, it becomes clear why the witness $\tilde{\mathcal{W}}_{4}$ requires only a moderate effort for its measurement: when comparing $\left|C_{6}\right\rangle\left\langle C_{6}\right|$ and $\left|C_{6}\right\rangle\left\langle C_{6}|+| \tilde{C}_{6}\right\rangle\left\langle\tilde{C}_{6}\right|$ in the standard basis, in the latter some off-diagonal terms are canceled. As we know from the analysis of the GHZ and the W state, such offdiagonal terms are typically difficult to measure.

Interestingly, other simple witnesses in the literature can also be viewed in this way. For instance, for the two-qubit singlet state $\left|\psi^{ \pm}\right\rangle=(|01\rangle \pm|10\rangle) / \sqrt{2}$ a witness would be $\mathcal{W}=\mathbb{1} / 2-\left|\psi^{-}\right\rangle\left\langle\psi^{-}\right|$which can be simplified to $\mathcal{W}^{\prime}=\mathbb{1} / 2-\left|\psi^{-}\right\rangle\left\langle\psi^{-}|+| \psi^{+}\right\rangle\left\langle\psi^{+}\right|=\left(\mathbb{1}+\sigma_{x} \otimes\right.$ $\left.\sigma_{x}+\sigma_{y} \otimes \sigma_{y}\right) / 2$, which is known from Ref. [10]. For the four-qubit cluster state $\left|C_{4}\right\rangle=(|0000\rangle+|0011\rangle+$ $|1100\rangle-|1111\rangle) / 2$ we can consider again the orthogonal state $\left|\tilde{C}_{4}\right\rangle=(-|0000\rangle+|0011\rangle+|1100\rangle+|1111\rangle) / 2$ and then the witness $\mathcal{W}^{\prime}=\mathbb{1} / 2-\left|C_{4}\right\rangle\left\langle C_{4}|+| \tilde{C}_{4}\right\rangle\left\langle\tilde{C}_{4}\right|$. This observable requires four measurements and has, in a different form, already been derived in Ref. [11].

Observation 3. For cluster states, one can derive sequences of witnesses, which require more and more measurements for an implementation, while becoming more and more robust to noise. Some of them can be improved by local filters, even if only few measurements have been done. Furthermore, a promising ansatz for a robust witness for a state $|\psi\rangle$ which requires only a moderate effort, is to consider $\mathcal{W}=\alpha \mathbb{1}-|\psi\rangle\langle\psi|+| \phi\rangle\langle\phi|$, where $|\phi\rangle$ is orthogonal to $|\psi\rangle$.

Let us finally discuss an example how one can derive entanglement witnesses beyond the projector based witnesses. We consider states in decoherence free subspaces. These are pure states $|\psi\rangle$, which are invariant under a simultaneous unitary rotation on all qubits, i.e. we have $(U)^{\otimes N}|\psi\rangle=e^{i \phi}|\psi\rangle$ (or, equivalently $\left[|\psi\rangle\langle\psi|,(U)^{\otimes N}\right]=$ $0)$. An example is the four-qubit singlet state [18]

$$
\left|\Psi_{4}\right\rangle=\frac{1}{\sqrt{3}}\left(|0011\rangle+|1100\rangle-\frac{1}{2}(|01\rangle+|10\rangle) \otimes(|01\rangle+|10\rangle)\right)
$$

A natural witness for this state would be $\mathcal{W}=3 / 41-$ $\left|\Psi_{4}\right\rangle\left\langle\Psi_{4}\right|$. It detects the state for $p>0.733$ and requires the measurement of 15 local settings [3].
In order to write down a simpler witness, note that due to the unitary invariance of $\left|\Psi_{4}\right\rangle$ we have $\left(\sigma_{k}\right)^{\otimes 4}\left|\Psi_{4}\right\rangle=$ $\left|\Psi_{4}\right\rangle$, for $k=x, y, z$. That is, observables of the type $g_{k}=$ $\left(\sigma_{k}\right)^{\otimes 4}$ are stabilizing operators of $\left|\Psi_{4}\right\rangle$. Therefore, they may be a good tool to construct entanglement witnesses. It should be noted, however, that $\left|\Psi_{4}\right\rangle$ is not the only common eigenstate of the $g_{k}$, the product of two singlet states, $|\phi\rangle=\left|\psi^{-}\right\rangle \otimes\left|\psi^{-}\right\rangle$is a different one.

In order to distinguish between the $\left|\Psi_{4}\right\rangle$ and the product of two-singlets, let us consider

$$
\begin{aligned}
& \mathcal{D}\left(\sigma_{k}\right)=\left(\sigma_{k}\right)^{\otimes 4}+3\left[\left(\sigma_{k}\right)^{\otimes 2}(\mathbb{1})^{\otimes 2}+(\mathbb{1})^{\otimes 2}\left(\sigma_{k}\right)^{\otimes 2}\right] \\
& -\frac{3}{2}\left[\mathbb{1}\left(\sigma_{k}\right)^{\otimes 2} \mathbb{1}+\mathbb{1} \sigma_{k} \mathbb{1} \sigma_{k}+\sigma_{k} \mathbb{1} \sigma_{k} \mathbb{1}+\sigma_{k}(\mathbb{1})^{\otimes 2} \sigma_{k}\right]
\end{aligned}
$$

Here, the coefficients have been chosen in such a way, that for the four-qubit singlet state all terms have the expectation value one, e.g. $3\left\langle\Psi_{4}\left|\left[\left(\sigma_{k}\right)^{\otimes 2}(\mathbb{1})^{\otimes 2}\right]\right| \Psi_{4}\right\rangle=1$, etc. Then we consider $\mathcal{Q}=\mathcal{D}\left(\sigma_{x}\right)+\mathcal{D}\left(\sigma_{y}\right)+\mathcal{D}\left(\sigma_{z}\right)$ For the singlet state we have $\left\langle\Psi_{4}|\mathcal{Q}| \Psi_{4}\right\rangle=21$. For a biseparable state $\varrho_{\mathrm{bs}}$, direct numerical optimization gives $\operatorname{Tr}\left(\varrho_{\mathrm{bs}} \mathcal{Q}\right) \leq 14.35$. Therefore, $\mathcal{W}=14.35 \cdot \mathbb{1}-\mathcal{Q}$ is a valid witness. This witness has interesting properties: It requires only three measurement settings (namely $\left.\left(\sigma_{k}\right)^{\otimes 4}, k=x, y, z\right)$ and detects noisy states for $p>0.683$. Therefore, despite of being simpler than the projector based witness, it is more robust to noise, and we have:

Observation 4. For states in decoherence-free subspaces, entanglement witnesses can be constructed from their stabilizing operators $g_{k}=\left(\sigma_{k}\right)^{\otimes N}$. These witnesses are independent of the projector based witness.

In conclusion, we have shown how to estimate the fidelity and detect the entanglement for several important families of states. Our constructions demonstrate that one can typically solve these problems with a small experimental effort. Therefore, our results will be useful for future experiments on multipartite entanglement.

We thank P. Hyllus, G. Tóth and W. Wieczorek for discussions. This work has been supported by the FWF, the EU (OLAQUI, SCALA, QICS, Marie Curie Excellence Grant) and the Alexander von Humboldt Foundation. This work has also been supported by NNSF, the CAS, and the NFRP of China.
[1] Z. Zhao et al., Nature (London) 430, 54 (2004); P.Walther et al., ibid. 434, 169 (2005); D. Leibfried et al., ibid. 438, 639 (2005); N. Kiesel et al., Phys. Rev. Lett. 95, 210502 (2005); ibid. 98, 063604 (2007); G. Vallone et al., ibid. 98, 180502 (2007); K. Chen et al., arXiv:0705.0174v1 [quant-ph].

[2] H. Häffner et al., Nature (London) 438, 643 (2005).

[3] M. Bourennane et al., Phys. Rev. Lett. 92, 087902 (2004).

[4] C.-Y. Lu et al., Nature Physics 3, 91 (2007).

[5] Technically speaking, a pure $N$-qubit state $|\psi\rangle$ is called biseparable, iff it can be written as $|\psi\rangle=|\alpha\rangle \otimes|\beta\rangle$ where $|\alpha\rangle$ and $|\beta\rangle$ are states on $M$ and $N-M$ qubits. A mixed state is biseparable, if it can be written as a convex combination of biseparable pure states, and a state which is not biseparable, is called genuine multipartite entangled. See also Refs. [3, 8].

[6] M. Horodecki, P. Horodecki, and R. Horodecki, Phys. Lett. A 223, 1 (1996); B. Terhal, Phys. Lett. A 271, 319 (2000); M. Lewenstein et al., Phys. Rev. A 62, 052310 (2000); D. Bruß et al., J. Mod. Opt. 49, 1399 (2002); O. Gühne and N. Lütkenhaus, Phys. Rev. Lett. 96, 170502 
(2006); S.J. van Enk, N. Lütkenhaus and H.J. Kimble, Phys. Rev. A 75, 052318 (2007); G. Tóth, J. Opt. Soc. Am. B 24, 275 (2007).

[7] B.M. Terhal, Theoret. Comput. Sci. 287, 313 (2002); O. Gühne et al., Phys. Rev. A 66, 062305 (2002).

[8] O. Gühne and P. Hyllus, Int. J. Theor. Phys. 42, 1001 (2003).

[9] G. Tóth and O. Gühne, Phys. Rev. Lett. 94, 060501 (2005).

[10] G. Tóth and O. Gühne, Phys. Rev. A 72, 022340 (2005).

[11] Y. Tokunaga et al,, Phys. Rev. A 74, 020301(R) (2006).

[12] L. Chen and Y.-X. Chen, Phys. Rev. A 76, 022330 (2007).

[13] R.D. Somma, J. Chiaverini, and D.J. Berkeland, Phys. Rev. A 74, 052302 (2006).

[14] For this calculation, the identities
$\sum_{k=1}^{N}(-1)^{k} \cos ^{m}(k \pi / N)=\left(1+(-1)^{N+m}\right) / 2$ (for $m<N)$ and $\sum_{k=1}^{N}(-1)^{k} \cos ^{m}(k \pi / N)=N / 2^{N-1}$ (for $m=N$ ) are of great help. See A. Prudnikov, Y. Brychkov and O. Maridev, Integrals and Series, Vol. 1, Taylor and Francis (1998), p. 640.

[15] The symbol $\lfloor x\rfloor$ denotes the largest integer which is smaller or equal $x$.

[16] W. Dür, G. Vidal, and I. Cirac, Phys. Rev. A 62, 062314 (2000).

[17] M. Hein, J. Eisert, and H.-J. Briegel, Phys. Rev. A 69, 062311 (2004).

[18] H. Weinfurter and M. Żukowski, Phys. Rev. A, 64, 010102 (2001); A. Cabello, Phys. Rev. A 75, 020301 (2007). 